\title{
A special symptom of olfactory dysfunction in coronavirus disease 2019: report of three cases
}

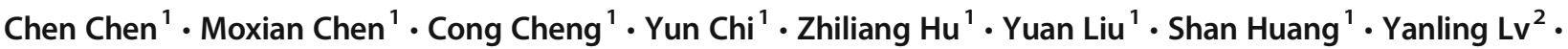

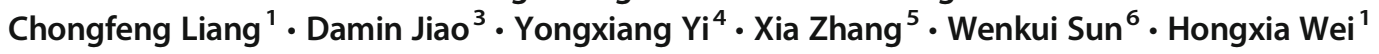

Received: 24 March 2020 / Revised: 18 April 2020 / Accepted: 29 April 2020 / Published online: 18 May 2020

(C) Journal of NeuroVirology, Inc. 2020

\begin{abstract}
Three patients of coronavirus disease (COVID-19) showed the symptoms of olfactory dysfunction. Clinical characteristics and treatment were retrospective analyzed. Olfactory disorders are uncommon symptoms of COVID-19 in China. Early diagnosis and intervention are keys to the recovery of olfactory disorders. Particular attention should be devoted to olfactory dysfunction.
\end{abstract}

Keywords COVID-19 · Olfactory dysfunction

\section{Introduction}

Since December 2019, when the first patient with a confirmed case of COVID-19 was reported in Wuhan, China, over 1,000, 000 patients with confirmed cases have been reported worldwide. It has been reported that the most common symptoms include fever, fatigue, dry cough, anorexia, myalgia, and dyspnea. Meanwhile, less common symptoms are nasal congestion, headache, runny nose, sore throat, and diarrhea (Wang et al. 2020). Although the number of clinical cases with olfactory dysfunction is gradually increasing abroad (Lechien et al. 2020), it has not been reported so far in China. We report 3 cases of new coronavirus pneumonia with olfactory dysfunction, two of which were accompanied by taste disorders.

Chen Chen and Moxian Chen contributed equally to this work.

Wenkui Sun

sunwenkui@njmu.edu.cn

Hongxia Wei

wghongxia@sina.com

1 Department of Infectious Disease, Nanjing Infectious Disease Center, The Second Hospital of Nanjing, Nanjing University of Chinese Medicine, \#1-1 Zhongfu Road, Nanjing 210003, Jiangsu, China

2 Department of Respiratory \& Critical Care Medicine, Nanjing Infectious Disease Center, The Second Hospital of Nanjing, Nanjing University of Chinese Medicine, Nanjing, Jiangsu, China
These cases will improve awareness of the symptoms and the treatment of the disease.

\section{Case report}

\section{Case 1}

It is female, age 18 , with a fever and dry cough since February 1, 2020. The symptoms quickly disappeared after being admitted to the hospital on February 3 for antiviral treatment with darunavir/cobicistat and arbidol. Since February 10, she noted complete anosmia. Due to no awareness, it is hard to know when this symptom appears. Subsequently, oral methylcobalamin tablets were

3 Department of Rheumatology and Immunology, Nanjing Infectious Disease Center, The Second Hospital of Nanjing, Nanjing University of Chinese Medicine, Nanjing, Jiangsu, China

4 Department of Hepatobiliary Surgery, Nanjing Infectious Disease Center, The Second Hospital of Nanjing, Nanjing University of Chinese Medicine, Nanjing, Jiangsu, China

5 Department of Tuberculosis, Nanjing Infectious Disease Center, The Second Hospital of Nanjing, Nanjing University of Chinese Medicine, Nanjing, Jiangsu, China

6 Department of Respiratory \& Critical Care Medicine, The First Affiliated Hospital of Nanjing Medical University, \#300 Guangzhou Road, Nanjing 210029, Jiangsu, China 
administered. As of February 21, the patient can smell irritating odors, such as alcohol, but the olfactory function has not yet returned to normal (Fig. 1).

\section{Case 2}

Female, age 26, with a sore throat and dry cough from February 7, 2020, was placed in an isolation room the next day. The antiviral therapy was arbidol and ribavirin. She recovered from these symptoms 5 days later. On February 9, the patient reported a sudden loss of sense of smell and taste simultaneously, accompanied by severe double-sided nasal congestion, runny nose, and severe headache, which were relieved after 2 days. But the anosmia and taste disorder did not improve. Methylcobalamin tablets were applied from February 14, and on February 15, a temporary intravenous drip of methylprednisolone $40 \mathrm{mg}$ was applied. The next day, the smell and taste improved and were restored to usual on February 24.

\section{Case 3}

Male, age 27, with muscle aches, fever and dry cough since 31 January 2020, was admitted to an isolation room on February 8 for antiviral treatment with lopinavir/ritonavir and arbidol. The patient had completely lost the sense of smell and taste on February 3, accompanied by slight nasal obstruction and mucus. Because he did not pay attention to the symptoms, he could not smell anything 10 days later. The doctor added a dose of methylcobalamin tablets to his treatment starting on February 14 . His olfaction and gustation had returned to normal on February 24.

\section{Discussion}

All 3 cases were young people. Olfactory dysfunction occurred, on average, 6 days after the onset of the first symptom related to COVID-19 and aggravated rapidly. The qRT-PCR tests showed positive detection to throat swab and negative to nasal mucosa swab. Specifically, all patients recovered rapidly from common symptoms of COVID-19. However, the median recovery time of olfactory dysfunction was 20 days reflecting the slow recovery of neurological function. Neither hematology examination nor cranial CT showed any significant abnormality, which excluded olfactory/taste disorders caused by innate diseases, drugs, trauma, intracranial occupancy, influenza, and para-flu viral infections.

Studies have shown that severe acute respiratory syndrome coronavirus 2 (SARS-CoV-2) has the typical characteristics of coronavirus and shares approximately $85 \%$ homology with severe acute respiratory syndrome coronavirus (SARS-CoV) (Gralinski and Menachery 2020). Moreover, coronavirus has neuropathic effects. There have been reports of human severe acute respiratory syndrome (SARS) with concomitant peripheral neuropathy in all four limbs or acute olfactory neuropath (Tsai et al. 2005; Hwang 2006). Cases 2 and 3 were accompanied by nasal obstruction and recovered rapidly. Inflammatory edema of the mucous membrane may be the main mechanism. However, the slow relief on olfactory dysfunction may be related to damage to the olfactory central nervous system.

Angiotensin-converting enzyme 2 (ACE2) is thought to be the main host cell receptor of SARS-CoV-2. The expression pattern of ACE2 in different organs indicates the potential high risk for SARS-CoV-2 infection and may be closely related to clinical manifestations (Zou et al. 2020). In particular, a high level of ACE2 expression was identified in numerous
Fig. 1 The course of symptoms and treatments

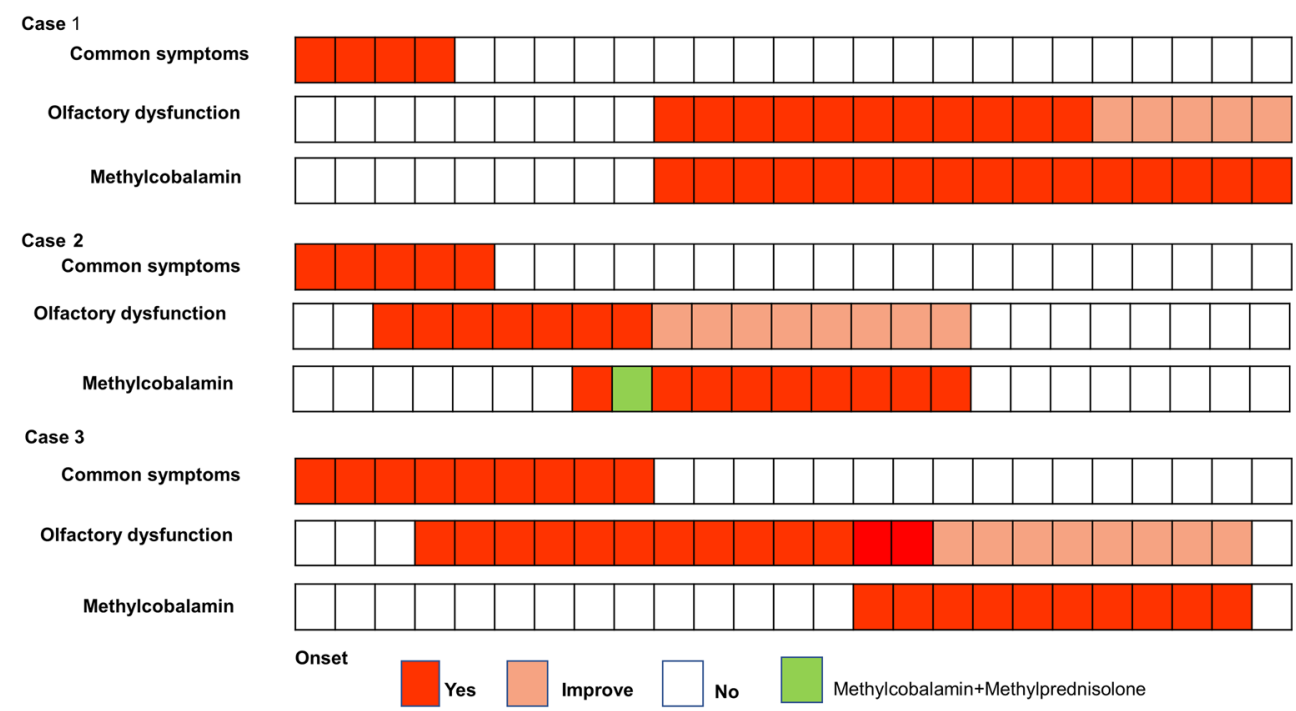


cell types, including esophageal upper and stratified epithelial cells, type II alveolar cells of lung, myocardial cells, absorptive enterocytes of the ileum and colon, cholangiocytes, kidney proximal tubule cells, and bladder urothelial cells (Zou et al. 2020). For example, high expression of ACE2 in the genitourinary system in patients with COVID-19 causes rare symptoms of orchitis. Thus, the accompanying taste disorders in these two cases may be also related to the expression level of ACE2 in the epithelial cells of the oral mucosa (Xu et al. 2020). The aforementioned examples provided a potential link between SARS-CoV-2 infection and olfactory disorders. However, the underlying mechanism requires further study. In addition, all three patients in this report were young people, and whether the expression level of ACE2 is related to age is currently unknown.

COVID-19 could be a special neuropathy that can cause permanent damage on human health. Additionally, intensive consultation and further detection are required in these cases according to the ignorance of uncommon symptoms, such as symptoms of neurological injury. Furthermore, there is no strong clinical evidence to show an effective clinical intervention, despite the use of corticosteroids or neurotrophic drugs (Harless and Liang 2016). Restricted by drug kinds, we chose methylcobalamin, which has the function of nerve nutrition, and methylprednisolone, which is thought to have a significant effect in treatments of olfactory dysfunction (Schriever et al. 2012). However, the number of cases is limited. The therapeutic efficacy needs to be confirmed in a larger cohort. Based on these three cases, early diagnosis and intervention would be the key to the recovery of olfactory dysfunction, particularly for young people.

Acknowledgments We gratefully acknowledge all those who contributed to this work, in particular, Nanjing Infectious Disease Center staff for their contributions to the clinical care of the patients. Also, we thank American Journal Experts for providing free language editing on this manuscript.

Authors' contributions Data collections were performed by Yuan Liu, Shan Huang, Yanling Lv, Chongfeng Liang, Damin Jiao and Chen Chen. The first draft of the manuscript was written by Chen Chen and Moxian Chen. All authors commented on previous versions of the manuscript. Critical revision of the manuscript was performed by Wenkui Sun and Hongxia Wei. All authors read and approved the final manuscript.
Funding information This work was supported by the National Science Foundation of China (NSFC 81770009).

\section{Compliance with ethical standards}

Conflict of interest The authors declare that they have no conflict of interest. All authors meet the ICMJE authorship criteria.

\section{References}

Gralinski LE, Menachery VD (2020) Return of the coronavirus: 2019$\mathrm{nCoV}$. Viruses 12:E135

Harless L, Liang J (2016) Pharmacologic treatment for postviral olfactory dysfunction: a systematic review. Int Forum Allergy Rhinol 6:760767

Hwang CS (2006) Olfactory neuropathy in severe acute respiratory syndrome: report of a case. Acta Neurol Taiwanica 15:26-28

Lechien JR, Chiesa-Estomba CM, De Siati DR, Horoi M, Le Bon SD, Rodriguez A, Dequanter D, Blecic S, El Afia F, Distinguin L, Chekkoury-Idrissi Y, Hans S, Delgado IL, Calvo-Henriquez C, Lavigne P, Falanga C, Barillari MR, Cammaroto G, Khalife M, Leich P, Souchay C, Rossi C, Journe F, Hsieh J, Edjlali M, Carlier R, Ris L, Lovato A, De Filippis C, Coppee F, Fakhry N, Ayad T, Saussez S (2020). Olfactory and gustatory dysfunctions as a clinical presentation of mild-to-moderate forms of the coronavirus disease (COVID-19): a multicenter European study. Eur Arch Otorhinolaryngol. 2020. doi: https://doi.org/10.1007/s00405-02005965-1. Online ahead of print

Schriever VA, Merkonidis C, Gupta N, Hummel C, Hummel T (2012) Treatment of smell loss with systemic methylprednisolone. Rhinology 50:284-289

Tsai LK, Hsieh ST, Chao CC, Chen YC, Lin YH, Chang SC, Chang YC (2005) Neuromuscular Disorders in Severe Acute Respiratory Syndrome. 61:1669

Wang D, Hu B, Hu C, Zhu F, Liu X, Zhang J, Wang B, Xiang H, Cheng Z, Xiong Y, Zhao Y, Li Y, Wang X, Peng Z (2020) Clinical characteristics of 138 hospitalized patients with 2019 novel coronavirusinfected pneumonia in Wuhan, China. JAMA 323:1061. https://doi. org/10.1001/jama.2020.1585

Xu H, Zhong L, Deng J, Peng J, Dan H, Zeng X, Li T, Chen Q (2020) High expression of ACE2 receptor of 2019-nCoV on the epithelial cells of oral mucosa. Int J Oral Sci 12:8

Zou X, Chen K, Zou J, Han P, Hao J, Han Z (2020). Single-cell RNA-seq data analysis on the receptor ACE2 expression reveals the potential risk of different human organs vulnerable to 2019-nCoV infection. Front Med

Publisher's note Springer Nature remains neutral with regard to jurisdictional claims in published maps and institutional affiliations. 